\title{
Echocardiographic study of myocardial work in patients with type 2 diabetes mellitus
}

\author{
Lisi Liao $^{1,2 \dagger}$, Bobo Shi ${ }^{1,2 \dagger}$, Zhimin Ding ${ }^{1,2+}$, Lixin Chen ${ }^{1,2}$, Fajin Dong ${ }^{1,2}$, Jian Li ${ }^{1,2}$, Yulin Zhong ${ }^{1,2}$ and \\ Jinfeng $X^{1,2^{*}}$
}

\begin{abstract}
Background: A noninvasive left ventricular (LV) pressure-strain loop (PSL) provides a new method to quantify myocardial work (MW) by combining global longitudinal strain (GLS) and LV pressure, which exerts potential advantages over traditional GLS. We studied the LV PSL and MW in patients with type 2 diabetes mellitus (T2DM).

Methods: This cross-sectional study included 201 subjects (54 healthy controls and 147 T2DM patients) who underwent complete two-dimensional echocardiography (2DE), including 2D speckle-tracking echocardiography (STE), as well as brachial artery pulse pressure measurement. The PSL was used to determine the global myocardial work index (GWI), global constructive work (GCW), global wasted work (GWW), and global work efficiency (GWE) of all study participants. The association between T2DM and LV function was evaluated according to these MW indices.

Results: The GLS was significantly lower in the T2DM group than in the control group $(P<0.001)$, indicating that the LV myocardium had been damaged, although the LV ejection fraction (LVEF) was still normal. The GWI and GWE were decreased $(P=0.022)$ and the GWW was increased $(P<0.001)$ in diabetic patients compared with controls, but the GCW was comparable in the two groups $(P=0.160)$. In all diabetic patients, age, body mass index, systolic blood pressure, smoking history, and LVEF were correlated with GWI, GWW and GWE.
\end{abstract}

Conclusions: The use of LV PSL is a novel noninvasive technique that could help to depict the relationship between LV myocardial damage and MW in patients with T2DM.

Keywords: Diabetes, Echocardiography, Left ventricular pressure-strain loop, Myocardial work

\section{Background}

Type 2 diabetes mellitus (T2DM) is a global epidemic. The global prevalence of diabetes in 2019 was estimated to be $9.3 \%$ (463 million) and is expected to increase to $10.2 \%$ ( 578 million) by 2030 and $10.9 \%$ (700 million) by 2045. T2DM accounts for approximately $90 \%$ of the total,

*Correspondence: xujinfeng@yahoo.com

†'Lisi Liao, Bobo Shi and Zhimin Ding contributed equally to this work

${ }^{1}$ Department of Ultrasound, First Affiliated Hospital of Southern

University of Science and Technology, Shenzhen Medical Ultrasound

Engineering Center, Shenzhen People's Hospital, 1017 Dongmen North

Road, Luohu District, Shenzhen 518020, Guangdong, China

Full list of author information is available at the end of the article with an estimated number of cases of approximately 520 million by 2030 and 630 million by $2045[1,2]$.

Studies have shown that diabetes can lead to the development of structural heart disease and heart failure (HF) through systemic, myocardial and cellular mechanisms [3]. A review of recent studies has detailed the underlying mechanisms of diabetes-related HF [4], emphasizing that hyperglycemia and hyperinsulinemia accelerate atherosclerosis through vascular smooth muscle cell (VSMC) proliferation and inflammation. In a recent study, Daniele Torella et al. showed that miR-29c overexpression and miR-204 inhibition fostered a reduction in the phenotypic switch of VSMCs in T2DM [5]. Diabetes is associated with atherogenic dyslipidemia, in which low-density 
lipoprotein (LDL) cholesterol particles are likely to cause atherosclerosis and are associated with endothelial dysfunction, which promotes leukocyte and platelet adhesion, thrombosis, inflammation, and coronary plaque ulceration [6]. Therefore, T2DM is a risk factor for cardiovascular diseases, such as myocardial ischemia and infarction [7]. At the same time, the risk of HF in diabetic patients was more than twice that in nondiabetic patients. Moreover, compared with patients without diabetes, patients with diabetes have worse cardiovascular outcomes, higher rates of hospitalization and a poorer prognosis [8]. In fact, tight glycemic control during acute coronary syndromes reduces the mortality rate and improves the prognosis of patients with and without diabetes $[9,10]$. Cardiovascular diseases worsen the quality of life of patients with T2DM and increase their medical costs. As a result, the early detection of cardiovascular disease in patients with T2DM is essential to improve outcomes in this population.

There are three kinds of cardiac abnormalities in diabetic heart disease: left ventricular (LV) systolic dysfunction, diastolic dysfunction and LV geometric changes. Echocardiography is the most commonly used method to examine the cardiac function of patients with DM. Determination of the ejection fraction (EF) and speckle tracking are commonly used to evaluate cardiac function. Although a decreased EF is considered a sign of HF in symptomatic patients, most patients with HF are still in the stage of EF preservation [11, 12], which cannot be efficiently detected by traditional echocardiographic parameters. The LV global longitudinal strain (GLS) is a load-dependent measurement of LV function; however, its accuracy in evaluating LV function under certain hemodynamic conditions is challenging [13]. Therefore, developing noninvasive technologies to evaluate myocardial lesions in diabetic patients is particularly important.

Myocardial work (MW) is a set of parameters related to afterload that overcomes the load dependence of the LVEF and LV strain. It is a dynamic noninvasive method that considers myocardial deformation and blood pressure (BP). This method was developed nearly 30 years ago, but it takes into account the invasive measurements obtained during cardiac catheterization, which is impractical in clinical practice. The global myocardial work index (GWI), global constructive work (GCW), global wasted work (GWW), and global work efficiency (GWE) are MW parameters that are derived from the analysis of the LV pressure-strain loop (PSL) combined with noninvasive $\mathrm{BP}$ and strain [14-17]. Although MW has shown great clinical potential, there have been few studies on LV MW in diabetic patients.

Therefore, the aim of this study was (1) to describe global indices of MW in healthy individuals and patients with T2DM; (2) to assess the correlation between MW and other basic and echocardiographic parameters; and (3) to evaluate the association of MW and adverse outcomes to determine the degree of myocardial damage in patients with T2DM and to monitor and protect myocardial function.

\section{Methods \\ Study population}

This was a cross-sectional study. Patients who underwent echocardiography in the Department of Ultrasound, Shenzhen People's Hospital from August 2020 to May 2021 were included.

The inclusion criteria were as follows: recent diagnosis ( $\leq 3$ months) of T2DM or poor glycemic control (glycosylated hemoglobin $(\mathrm{HbA} 1 \mathrm{c}) \geq 6.5 \%$ and fasting plasma glucose $\geq 7.0 \mathrm{mmol} / \mathrm{L}$ ).

The exclusion criteria were as follows: HF; coronary artery disease (coronary artery disease was identified by medical history, coronary computed tomography angiography, echocardiography, invasive coronary imaging, electrocardiography, cardiopulmonary exercise test, and clinical symptoms of coronary artery disease); moderate or above valvular regurgitation disease; atrial fibrillation; tumor; liver cirrhosis or renal failure; hypertension with poor BP control; and poor echocardiogram image quality.

Basic data were obtained from all participants and included sex, age, height, weight, body mass index (BMI), smoking history, BP, T2DM duration and laboratory examination data [fasting blood glucose $(\mathrm{mmol} / \mathrm{L})$, triglyceride $(\mathrm{mmol} / \mathrm{L})$, high-density lipoprotein (HDL, $\mathrm{mmol} / \mathrm{L})$, LDL $(\mathrm{mmol} / \mathrm{L})$, total cholesterol $(\mathrm{mmol} / \mathrm{L})$, creatinine $(\mu \mathrm{mol} / \mathrm{L})$, and $\mathrm{HbA1c}(\%)]$. T2DM was diagnosed according to current clinical guidelines. Data related to drug use were obtained from all participants in the study. The study was approved by the local ethics committee of our hospital, and written informed consent was obtained from all participants.

\section{BP measurement}

BP measurement for each patient was performed in a quiet $25{ }^{\circ} \mathrm{C}$ room after a 10 -min supine rest. We measured the brachial artery BP and heart rate in the right arm with an automatic digital sphygmomanometer (OMRON, Kyoto, Japan). BP was measured before and immediately after echocardiography, and the mean value was used for statistical analysis.

\section{Echocardiography}

Echocardiography was performed with a model E95 ultrasound machine (GE Healthcare). All parameters were the average of three consecutive cardiac cycles. The left atrial dimension (LAD), LV end-diastolic dimension 
(LVEDD), LV end-systolic dimension (LVESD), LV enddiastolic volume (LVEDV), LV end-systolic volume (LVESV), interventricular septum (IVS) thickness, posterior wall thickness (PWT), and LV stroke volume (LVSV) were obtained according to the guidelines of the American Society of Echocardiography [18]. The LVEF was evaluated by the biplane method, and Doppler blood flow and tissue Doppler velocity imaging were used to obtain the $\mathrm{E}$ wave $(\mathrm{cm} / \mathrm{s})$, A wave $(\mathrm{cm} / \mathrm{s}), E / A$ ratio, septal $\mathrm{e}^{\prime}$ wave $(\mathrm{m} / \mathrm{s})$, lateral $\mathrm{e}^{\prime}$ wave $(\mathrm{m} / \mathrm{s})$, and $\mathrm{E} / \mathrm{e}^{\prime}$ ratio.

\section{LV strain analysis and MW analysis}

Three consecutive cardiac cycles were collected for two-dimensional (2D) strain analysis [18]. Q-analysis (echoPAC version 202, GE Healthcare) was used to evaluate 2D strain. Data were collected from three apical views (3, 4 and 2 chambers). The GLS of the LV myocardium was evaluated on the apical view. The performing physician verified the endocardial contour automatically tracked by the software and adjusted the region of interest to confirm that all images included the entire LV wall thickness. The left ventricle was divided into 17 segments to calculate the longitudinal strain.

MW was evaluated using the same software. After calculating the GLS of the left ventricle, the brachial artery $\mathrm{BP}$ was entered, and the valve opening and closing time was determined by echocardiography $[19,20]$. The timing of valve opening and closing was determined by spectral Doppler imaging at the mitral and aortic valve levels. The software provides a noninvasive PSL by integrating GLS, BP and valve opening and closing timing data. The annular area, as does the GWI, corresponds to the total work from mitral valve closure to mitral valve opening in the LV pressure-strain annular area. In addition, other indices of MW were calculated (Fig. 1), as follows: GCW (work done by systolic LV shortening and diastolic LV lengthening); GWW (work done by systolic LV lengthening and diastolic LV shortening); and GWE (effective work divided by the sum of effective work and ineffective work). All studies were analyzed by two ultrasound physicians (LLS and SBB) blinded to the clinical and laboratory data (double-blinded analysis).

\section{Statistical analysis}

Continuous variables are represented as the means \pm standard deviations and were compared using independent sample t-tests. Age, BMI and systolic BP (SBP) were used as covariates, and multivariate correlation analysis was used to evaluate the relationship of basic, laboratory and echocardiographic parameters with MW parameters. Interobserver and intragroup correlation coefficients (ICCs) were used to assess repeatability. $P<0.05$ was considered statistically significant.

\section{Results}

\section{Study population}

This study population consisted of 201 individuals: 147 patients with T2DM (mean age: $51.35 \pm 12.06$ years; $66 \%$ male) and 54 healthy controls (mean age: 48 . $33 \pm 9.88$ years; $53 \%$ male). The clinical characteristics of the patients in the two groups are shown in Table 1. There was no significant difference in age or sex between the two groups, but there was a significant difference in BMI, smoking history, SBP and diastolic BP (DBP) $(P<0.05)$. In terms of laboratory parameters, there were significant differences in fasting blood glucose, HDL, LDL, triglycerides, and HbAlc between the groups; the differences in fasting blood glucose, $\mathrm{HDL}$, triglycerides and $\mathrm{HbA1c}$ were all significant with $P<0.01$.

\section{Results of traditional echocardiography}

Table 2 presents the echocardiographic characteristics of the diabetic patients and healthy controls. The LAD was greater in the diabetic patients than in the controls (32. $93 \pm 3.85$ vs. $30.14 \pm 3.89 \mathrm{~mm} ; P<0.001$ ), and the IVS thickness and PWT were greater $(10.48 \pm 1.46$ vs. $8.53 \pm 1.25 \mathrm{~mm}, 9.94 \pm 1.59$ vs. $9.94 \pm 1.59 ; P<0.001$ ). There was a significant difference in the $E / A$ ratio and $\mathrm{E} / \mathrm{e}$ ' ratio between the control and T2DM groups $(1.42 \pm 0.40$ vs. $0.88 \pm 0.32 ; 7.09 \pm 1.69$ vs. $9.64 \pm 3.24)$. Although the LV volume of diabetic patients was slightly lower and LV diastolic function was more impaired, there was no significant difference in the LVEDD, LVESD, LVEDV, LVESV, or LVEF between the diabetic patients and controls.

\section{GLS and LV MW}

Table 3 summarizes the LV GLS and MW data. Compared with the controls, the LV GLS was significantly impaired in the diabetic patients $(-16.820 \pm 2.59 \%$ vs. $-19.13 \pm 1.72 \% ; P<0.001)$. The GWI in the control and T2DM groups was $1692.86 \pm 349.63$ vs. $1814.50 \pm 270.50 \mathrm{mmHg} \% \quad(P<0.05)$. There was no significant difference in the GCW between the control and T2DM groups (1998. $760 \pm 362.53$ vs. $2075.13 \pm 296.14 \mathrm{mmHg} \%, P=0.160$ ), and the GWW was significantly greater in the T2DM group than in the control group $(140 \pm 87.63 \mathrm{mmHg} \%$ [IQR: $126.67-$ $155.24 \mathrm{mmHg} \%$ ] vs. $69.22 \pm 31.49 \mathrm{mmHg} \%$ [IQR: $60.63-77.82 \mathrm{mmHg} \%] ; P<0.001)$. This condition led to a decrease in GWE; the GWE in the T2DM group was 92.26\% $\pm 28 \%$ (IQR: 91.56-92.69\%), while that in the control group was $96.07 \% \pm 1.52 \%$ (IQR: $95.66-96.49 \%$ ) $(P<0.001)$. 


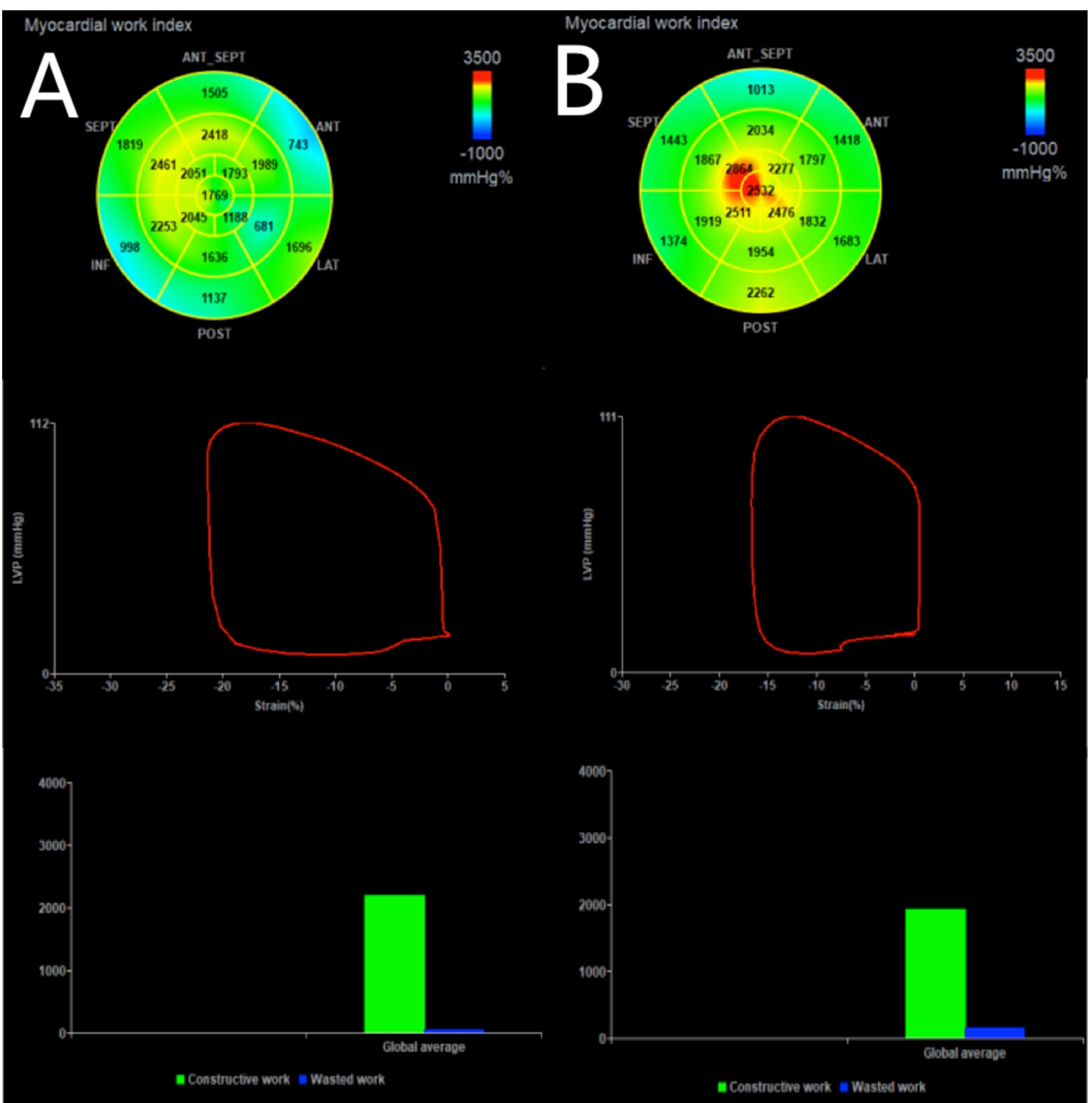

Fig. 1 (Top panel) Seventeen-segment bull's-eye representation of MW index (GWI) showing areas of negative work in blue, normal in green, and red indicating areas of high MW; (Middle panel) LV PSL; (Bottom panel) Green bar shows constructive work; Blue bar shows wasted work. Examples of patients within: A control group; B T2DM

\section{Correlation between MW and other parameters}

GWI was correlated with triglycerides $(\beta=-0.13$, $P<0.05)$, sex $(\beta=-0.19, P<0.01)$, age $(\beta=0.19, P<0.01)$, BMI $(\beta=-0.18, P=0.01)$, smoking history $(\beta=-0.28$, $P<0.01)$, SBP $(\beta=0.34, P<0.01)$, fasting blood glucose $(\beta=-0.271, P<0.01)$, HDL $(\beta=0.20, P<0.01)$, and LVEF $(\beta=-0.23, P=0.001$, Table 4$)$.

GWW was correlated with BMI $(\beta=-0.15, P<0.05)$, smoking history $(\beta=-0.17, P<0.05)$, LVESV $(\beta=0.166$, $P<0.05)$, age $(\beta=0.28, P<0.01)$, SBP $(\beta=0.40, P<0.01)$, DBP $(\beta=0.20, P<0.01)$, LAD $(\beta=-0.23, P=0.001)$, IVS thickness $(\beta=0.40, P<0.01)$, LV PWT $(\beta=0.36, P<0.01)$, E/A ratio $(\beta=-0.33, P<0.01), E / \mathrm{e}^{\prime}$ ratio $(\beta=-0.27$, $P<0.05), \operatorname{LV}$ PWT $(\beta=0.36, P<0.01)$, and LVEF $(\beta=0.53$, $P<0.01)$.

GWE was correlated with LVEDV $(\beta=-0.14$, $P<0.05), \quad$ creatinine $\quad(\beta=-0.17, \quad P<0.05), \quad$ BMI $(\beta=-0.23, P=0.001)$, age $(\beta=-0.22, P<0.01)$, smoking history $(\beta=-0.485, P<0.01), \operatorname{SBP}(\beta=-0.33$, $P<0.01)$, DBP $(\beta=-0.23, P=0.001)$, fasting blood glucose $(\beta=-0.20, P<0.01)$, HDL $(\beta=0.18, P<0.01)$, LAD $\quad(\beta=-0.28, \quad P<0.01), \quad$ LVESD $\quad(\beta=-0.21$, 
Table 1 Baseline characteristics of two groups

\begin{tabular}{lllr}
\hline & Controls $(\mathbf{n}=\mathbf{5 4})$ & T2DM $(\mathbf{n}=\mathbf{1 4 7})$ & $\boldsymbol{P}$ value \\
\hline Age (years) & $48.33 \pm 9.88$ & $51.35 \pm 12.06$ & 0.101 \\
Male (\%) & 53 & 66 & 0.075 \\
BMI (kg/m $\left.{ }^{2}\right)$ & $22.93 \pm 3.33$ & $27.04 \pm 5.59$ & $<0.001^{* *}$ \\
SBP $(\mathrm{mmHg})$ & $130.01 \pm 11.20$ & $132.03 \pm 18.67$ & $<0.001^{* *}$ \\
DBP (mmHg) & $76.05 \pm 8.10$ & $83.15 \pm 10.80$ & $<0.001^{* *}$ \\
Smoker (\%) & 1.8 & 38 & $<0.001^{* *}$ \\
Fasting glucose level (mmol/L) & $5.02 \pm 0.61$ & $7.75 \pm 2.61$ & $<0.001^{* *}$ \\
HbA1c (\%) & $5.46 \pm 0.58$ & $9.96 \pm 8.09$ & $<0.001^{* *}$ \\
Triglycerides (mmol/L) & $1.40 \pm 0.80$ & $2.31 \pm 2.31$ & $0.005^{* *}$ \\
TC (mmol/) & $5.02 \pm 0.61$ & $4.80 \pm 1.33$ & 0.245 \\
Scr (umol/L) & $71.93 \pm 16.86$ & $80.30 \pm 38.15$ & 0.122 \\
HDL (mmol/L) & $1.31 \pm 0.36$ & $1.07 \pm 0.30$ & $<0.001^{* *}$ \\
LDL (mmol/L) & $3.10 \pm 0.64$ & $2.75 \pm 0.98$ & $0.016^{*}$ \\
\hline
\end{tabular}

T2DM, type 2 diabetes mellitus; BMI, body mass index; SBP, systolic blood pressure; DBP, diastolic blood pressure; HbA1c, glycosylated hemoglobin; TC, Triglycerides; Scr, Serum creatinine; HDL, High density lipoprotein; LDL, Low density lipoprotein

${ }^{*} P<0.01$

$* * P<0.05$

Table 2 Echocardiographic parameters of left ventricular structure and function in the study population

\begin{tabular}{lllc}
\hline & Controls $(\mathbf{n}=\mathbf{5 4})$ & T2DM $(\mathbf{n}=\mathbf{1 4 7})$ & P value \\
\hline LAD $(\mathrm{mm})$ & $30.14 \pm 3.89$ & $32.93 \pm 3.85$ & $<0.001^{* *}$ \\
LVEDD $(\mathrm{mm})$ & $46.29 \pm 4.90$ & $45.46 \pm 5.11$ & 0.306 \\
LVESD $(\mathrm{mm})$ & $29.11 \pm 4.14$ & $28.88 \pm 4.11$ & 0.730 \\
IVS $(\mathrm{mm})$ & $8.52 \pm 1.25$ & $10.28 \pm 1.46$ & $<0.001^{* *}$ \\
PWT $(\mathrm{mm})$ & $8.29 \pm 1.32$ & $9.94 \pm 1.59$ & $<0.001^{* *}$ \\
LVEDV $(\mathrm{mL})$ & $100.59 \pm 24.89$ & $96.62 \pm 25.72$ & 0.330 \\
LVESV $(\mathrm{mL})$ & $32.14 \pm 10.66$ & $21.93 \pm 12.19$ & 0.677 \\
LVSV $\left(\mathrm{mL} / \mathrm{m}^{2}\right)$ & $67.88 \pm 16.96$ & $63.58 \pm 17.70$ & 0.125 \\
LVEF $(\%)$ & $67.69 \pm 5.28$ & $66.18 \pm 6.13$ & 0.062 \\
E/A ratio & $1.42 \pm 0.40$ & $0.88 \pm 0.32$ & $<0.001^{* *}$ \\
E/e' & $7.09 \pm 1.69$ & $9.64 \pm 3.24$ & $<0.001^{* *}$ \\
LVDF, normal & $54 / 54$ & $49 / 147$ & $<0.001^{* *}$
\end{tabular}

T2DM, type2 diabetes mellitus; LAD, left atrial dimension; LVEDD, left ventricular end-diastolic dimension; LVESD, left ventricular end-systolic dimension; IVS, interventricular septum; PWT, posterior wall thinkness, LVEDV, left ventricular end-diastolic volume; LVESV, left ventricular end-systolic volume; LVSV, left ventricular stroke volume; LVEF, left ventricular ejection fraction; $A$, late diastolic mitral flow (pulse Doppler); E, early diastolic mitral flow (pulse Doppler); mitral annulus(tissue Doppler); $\mathrm{e}^{\prime}$, average of the peak early diastolic relaxation velocity of the septal and lateral; LVDF, left ventricular diastolic function

**P $P 0.01$

${ }^{*} P<0.05$

$P<0.01)$, IVS thickness $(\beta=-0.41, P<0.01)$, PWT $(\beta=-0.40, \quad P<0.01), \quad$ LVESV $\quad(\beta=-0.26, \quad P<0.01)$, LVEF $(\beta=-0.23, \quad P=0.001), \quad \mathrm{E} / \mathrm{A}$ ratio $(\beta=0.35$, $P<0.01)$, E/e' ratio $(\beta=-0.27, P<0.01)$, and LVEF $(\beta=-0.55, P<0.01)$. Age, BMI, and SBP were used as
Table 3 speckle-tracking and myocardial work assessment of left ventricular function in the study population

\begin{tabular}{lccc}
\hline & Controls $(\mathbf{n}=\mathbf{5 4})$ & T2DM $(\mathbf{n}=\mathbf{1 4 7})$ & $\boldsymbol{P}$ value \\
\hline GLS, \% & $-19.13 \pm 1.73$ & $-16.82 \pm 2.59$ & $<0.001^{* *}$ \\
Myocardial work & & & \\
GWI (mmHg\%) & $1814.50 \pm 270.50$ & $1692.86 \pm 349.63$ & $0.022^{*}$ \\
GCW (mmHg\%) & $2075.13 \pm 269.14$ & $1998.76 \pm 362.53$ & 0.160 \\
GWW (mmHg\%) & $69.22 \pm 31.49$ & $140.96 \pm 87.63$ & $<0.001^{* *}$ \\
GWE (\%) & $96.07 \pm 1.52$ & $92.26 \pm 4.28$ & $<0.001^{* *}$ \\
\hline
\end{tabular}

T2DM, type2 diabetes mellitus; GLS, left ventricular global longitudinal strain; GWI, global myocardial work index; GCW, global constructive work; GWW, global wasted work; GWE, global myocardial work efficiency

${ }^{*} P<0.01$

${ }^{*} P<0.05$

covariates after correction, and there were still significant differences.

T2DM duration was associated with $\mathrm{E} / \mathrm{A}$ ratio $(\beta=-0.31, P<0.01), E / e^{\prime}$ ratio $(\beta=0.45, P<0.01), L V$ diastolic function $(\beta=0.36, P<0.01)$, GWI $(\beta=0.19$, $P<0.05)$ and GCW $(\beta=0.25, P<0.01)$. Moreover, there were no correlations with other echocardiographic parameters.

\section{Intraobserver and interobserver variability of $\mathrm{MW}$ parameters}

The interclass correlation coefficients (ICCs) for interobserver variability were as follows: GLS, 0.97 (95\% CI: 0.96-0.99; $P<0.01$ ); GWI, 0.95 (95\% CI: 0.90-0.97; 
Table 4 multivariate analyses of echocardiographic parameters and myocardial function parameters in patients with diabetes mellitus

\begin{tabular}{|c|c|c|c|c|c|c|}
\hline & \multicolumn{2}{|c|}{ GWI (mmHg\%) } & \multicolumn{2}{|c|}{ GWW (mmHg\%) } & \multicolumn{2}{|l|}{ GWE (\%) } \\
\hline & $\beta$ & $P$ & $\beta$ & $P$ & $\beta$ & $P$ \\
\hline Age (years) & 0.197 & $0.005^{* *}$ & 0.282 & $<0.001^{* *}$ & -0.221 & $0.002^{* *}$ \\
\hline Male (\%) & -0.188 & $0.007^{* *}$ & 0.004 & 0.954 & -0.069 & 0.327 \\
\hline $\mathrm{BMI}\left(\mathrm{kg} / \mathrm{m}^{2}\right)$ & -0.181 & $0.010^{* *}$ & 0.156 & $0.027^{*}$ & -0.238 & $0.001^{* *}$ \\
\hline Smoker & -0.281 & $<0.001^{* *}$ & 0.170 & $0.016^{*}$ & 0.485 & $<0.001^{* *}$ \\
\hline $\mathrm{SBP}(\mathrm{mmHg})$ & 0.343 & $<0.001^{* *}$ & 0.409 & $<0.001^{* *}$ & -0.333 & $<0.001^{* *}$ \\
\hline T2DM duration (years) & 0.198 & $0.016^{*}$ & 0.062 & 0.459 & 0.018 & 0.832 \\
\hline $\mathrm{DBP}(\mathrm{mmHg}$ & 0.025 & 0.724 & 0.206 & $0.003^{* *}$ & -0.231 & $0.001^{* *}$ \\
\hline Fasting glucose level (mmol/L) & -0.271 & $<0.001^{* *}$ & 0.132 & 0.063 & -0.204 & $0.004^{* *}$ \\
\hline $\mathrm{HbA1c}(\%)$ & -0.041 & 0.572 & 0.097 & 0.174 & -0.099 & 0.165 \\
\hline Triglycerides (mmol/L) & -0.139 & $0.049^{*}$ & 0.043 & 0.549 & -0.118 & 0.098 \\
\hline $\mathrm{TC}(\mathrm{mmol} / \mathrm{L})$ & 0.018 & 0.799 & -0.014 & 0.841 & 0.007 & 0.926 \\
\hline Scr (umol/L) & -0.026 & 0.717 & 0.081 & 0.259 & -0.179 & $0.012^{*}$ \\
\hline $\mathrm{HDL}(\mathrm{mmol} / \mathrm{L})$ & 0.203 & $0.004^{* *}$ & -0.103 & 0.146 & 0.189 & $0.007^{* *}$ \\
\hline $\mathrm{LDL}(\mathrm{mmol} / \mathrm{L})$ & 0.090 & 0.207 & -0.093 & 0.190 & 0.107 & 0.134 \\
\hline $\mathrm{LAD}(\mathrm{mm})$ & 0.006 & 0.933 & 0.234 & $0.001^{* *}$ & -0.282 & $<0.001^{* *}$ \\
\hline LVEDD (mm) & 0.009 & 0.901 & 0.105 & 0.139 & -0.135 & 0.057 \\
\hline $\operatorname{LVESD}(\mathrm{mm})$ & -0.094 & 0.187 & 0.132 & 0.061 & -0.210 & $0.003^{* *}$ \\
\hline IVS (mm) & -0.065 & 0.362 & 0.404 & $<0.001^{* *}$ & -0.415 & $<0.001^{* *}$ \\
\hline PWT (mm) & -0.127 & 0.072 & 0.361 & $<0.001^{* *}$ & -0.401 & $<0.001^{* *}$ \\
\hline LVEDV (mL) & 0.002 & 0.979 & 0.117 & 0.099 & -0.148 & $0.037^{*}$ \\
\hline LVESV (mL) & -0.132 & 0.062 & 0.166 & $0.018^{*}$ & -0.260 & $<0.001^{* *}$ \\
\hline $\operatorname{LVSV}\left(\mathrm{mL} / \mathrm{m}^{2}\right)$ & 0.069 & 0.332 & 0.052 & 0.464 & -0.032 & 0.651 \\
\hline LVEF (\%) & 0.237 & $0.001^{* *}$ & -0.133 & $0.060^{*}$ & 0.233 & $0.001^{* *}$ \\
\hline E/A ratio & 0.053 & 0.452 & -0.339 & $<0.001^{* *}$ & 0.350 & $<0.001^{* *}$ \\
\hline E/e' ratio & 0.126 & 0.075 & 0.275 & $<0.001^{* *}$ & -0.270 & $<0.001^{* *}$ \\
\hline LVDF & -0.059 & 0.409 & 0.533 & $<0.001^{* *}$ & -0.552 & $<0.001^{* *}$ \\
\hline
\end{tabular}

T2DM, type2 diabetes mellitus; BMI, body mass index; SBP, systolic blood pressure; DBP, diastolic blood pressure; HbA1c, glycosylated hemoglobin; TC, Triglycerides; Scr, Serum creatinine; HDL, High density lipoprotein; LDL, Low density lipoprotein; LAD, left atrial dimension; LVEDD, left ventricular end-diastolic dimension; LVESD, left ventricular end-systolic dimension; IVS, interventricular septum; PWT, posterior wall thickness; LVEDV, left ventricular end-diastolic volume; LVESV, left ventricular end-systolic volume; LVSV, left ventricular-stroke volume; LVEF, left ventricular ejection fraction; A, late diastolic mitral flow(pulse Doppler); E, early diastolic mitral flow(pulse Doppler); $\mathrm{e}^{\prime}$, mitral annulus(tissue Doppler); average of the peak early diastolic relaxation velocity of the septal and lateral; LVDF, left ventricular diastolic function

$* * 0.01$

${ }^{*} P<0.05$

$P<0.01$ ); GCW, 0.93 (95\% CI: 0.89-0.96; $P<0.01$ ); GWW, 0.82 (95\% CI: $0.68-0.94 ; P<0.01)$; and GWE, 0.88 (95\% CI: 0.55-0.96; $P<0.01)$.

The ICCs for intraobserver variability were as follows: GLS, 0.98 (95\% CI: 0.92-0.99; $P<0.01)$; GWI, 0.94 (95\% CI: 0.85-0.96; $P<0.01$ ); GCW, 0.91 (95\% CI: 0.83-0.95; $P<0.01$ ); GWW, 0.82 (95\% CI: 0.55-0.90; $P<0.05$ ); and GWE, 0.88 (95\% CI: 0.67-0.94; $P<0.01$ ).

\section{Discussion}

The main findings of this study are as follows: (1) The GLS was lower in the T2DM group than in the control group, indicating that the myocardial contractile function of diabetic patients had changed while the LVEF was preserved. (2) Compared with the control group, the GWI was significantly reduced in the T2DM group, but the GCW showed no significant difference, and the GWW was significantly greater in the T2DM group, which led to a decrease in the GWE in diabetic patients. (3) Age, BMI, SBP, smoking history, and LVEF were associated with GWI, GWW, and GWE in patients with diabetes.

Cardiovascular disease is the main cause of death in T2DM [21]. Diabetes can cause major vascular complications (cardiovascular disease) and microvascular complications (e.g., diabetic nephropathy, diabetic retinopathy, and neuropathy), which increase mortality, blindness, and renal failure in diabetic patients [22]. Therefore, 
some echocardiographic methods have been proposed to better evaluate the LV function of diabetic patients. In the past few years, determination of the LV GLS from strain tracking has become a daily measurement modality to evaluate LV function in diabetic patients and has shown a good correlation with histology-confirmed myocardial fibrosis [23, 24]. The GLS was lower in the T2DM group than in the control group, indicating that patients with diabetes have impaired myocardial contractility even when the LVEF is in the normal range. A decreased GLS is also common in asymptomatic diabetic patients, although the incidence is lower than that of diastolic dysfunction [11]. An abnormal GLS is related to the development of HF, mortality, and LV remodeling [12]. The reduced strain rate is a sign of contractility reduction, and the underlying causes include insulin signal changes, apoptosis, and necrosis leading to the loss of contractile cells and stress on the endoplasmic reticulum [25].

LV GLS is still dependent on load, which may be a limitation in the event of changes in hemodynamic conditions. Myocardial function has been introduced as a new LV function parameter that takes into account LV deformation and LV afterload. Based on noninvasive LV pressure (BP) measurement, the LV PSL was constructed. Compared with the traditional GLS method, this approach combines measurement of the myocardial deformation and stress load and has advantages [14, 26, 27]. Russell et al. also validated this method by invasive LV pressure measurements, and the LV PSL area was strongly correlated with myocardial metabolism during positron emission tomographic assessment [28].

The GWI, GCW, GWW, and GWE in the control group were consistent with the normal values obtained in the European Association of Cardiovascular Imaging (EACVI) Normal Reference Ranges for Echocardiography (NORRE) study. Our study also confirms the results of other previous studies [29]. In this study, the GWI was significantly lower in the T2DM group than in the control group, and while there was no difference in the GCW, the GWW was significantly increased, which led to a decrease in the GWE in the T2DM group. It is suggested that even if the LVEF is preserved, the GWI has already decreased, the GWW has significantly increased, and the GWE has decreased. LV dysfunction can occur in the early stage of myocardial impairment in diabetic patients. The hypothesis of diabetic myocardial disease includes changes in myocardial calcium homeostasis, mitochondrial dysfunction, oxidative stress, direct glucotoxicity, myocardial fibrosis, disorder of myocardial matrix use, and myocardial lipid accumulation [30, 31]. Specifically, T2DM is related to myocardial fibrosis or collagen content and myocardial stiffness [32,33]. Fibrosis of the endocardium, which causes focal fibrosis and low perfusion caused by the redistribution of myocardial blood flow due to its contraction, is impaired by myocardial deformation [34], which leads to a decrease in the GWI, an increase in the GWW, and subsequently a decrease in the GWE.

In this study, there was a significant difference in smoking among the baseline data between the control and T2DM groups. After multivariate regression analysis, smoking was significantly correlated with the GWI, GWW and GWE, indicating that smoking impairs myocardial function. There is evidence that nicotine can cause direct damage to the structure and function of the heart even in patients without atherosclerosis or other chronic diseases related to the risk of cardiovascular disease [35], and the amount of smoking is positively correlated with the degree of impairment of cardiac systolic function [36]. In the Multi-Ethnic Study of Atherosclerosis (MESA) study, it was found that the LV mass and geometry were associated with the occurrence of HF, stroke and coronary heart disease. Smoking can increase the LV mass/volume ratio and lead to a poor prognosis in patients with coronary heart disease [37].

In our study, SBP was significantly correlated with GWI, GWW and GWE, and DBP was significantly correlated with GWW and GWE, indicating that hypertension is associated with impaired myocardial function. Essential hypertension and T2DM are two common chronic diseases. T2DM can aggravate LV diastolic dysfunction in patients with essential hypertension $[38,39]$.

A recent study showed that subclinical myocardial dysfunction can be detected by real-time three-dimensional echocardiography (RT-3DE) in T2DM patients with poor glycemic control and that myocardial dysfunction is related to the duration of diabetes and level of $\mathrm{HbA1c}$ [40]. However, in our study, HbA1c showed no significant association with any echocardiographic parameter or MW index. It is speculated that this might be due to the small sample size of our study.

Gulsin GS et al. found that in asymptomatic workingage adults with T2DM, after weight loss, blood glucose control improved LV diastolic function, concentric LV remodeling and aortic stiffness, which provides the possibility that LV function in T2DM patients may be improved once metabolic control is obtained [41].

Recent data on diabetic patients showed that the use of either a glucagon-like peptide-1 receptor agonist (liraglutide) alone or in combination with a sodium-glucose cotransporter-2 inhibitor (empagliflozin) increased a number of cardiac work indicators in patients after 4 months of treatment and reduced cardiac failure compared to baseline $(P=0.041$ and $P<0.001$, respectively). All patients had an increase of MW index after 12 months of treatment [42]. It is suggested that LV 
myocardial function can be helpful in evaluating the myocardial condition of diabetic patients before and after treatment.

The introduction of cardiac parameters in the routine assessment of T2DM may improve the detection rate of early-stage heart disease in these patients. This is of particular importance in diabetic patients, as cardiovascular disease may change with the course of diabetes, use of medication, and level of blood glucose control. This approach could serve as a better tool for clinicians to improve the follow-up of LV function in these patients and assess the potential effects of different treatments. In addition, it may represent a new risk stratification tool for assessing the prognosis of patients with T2DM.

\section{Limitations}

There are several limitations to this study. First, the sample size was relatively small. In addition, the average age of the subjects was approximately 50 years. Thus, the generalization of our findings to other populations may require further confirmation. Second, we excluded some elderly patients upon speckle-tracking analysis failure, which may contribute to the risk of reporting bias. Third, we selected only patients with poorly controlled plasma glucose levels, leading to an increased risk of selection bias in this study. Finally, the study followed a cross-sectional design. Future studies with larger sample sizes following a cohort or randomized controlled trial design are needed to confirm our findings.

\section{Conclusions}

This study shows that the LV PSL method could be used to evaluate myocardial dysfunction in T2DM patients in the early stage. The GWW of the patients was significantly increased, and the GWI and GWE were decreased, indicating that myocardial contractile function was damaged even while the LVEF was preserved. Age, BMI, SBP, smoking history and LVEF were all independently related to MW. Future studies with larger sample sizes evaluating the prognostic impact of MW on cardiovascular outcomes in diabetes are needed.

\footnotetext{
Abbreviations

T2DM: Type 2 diabetes mellitus; LV: Left ventricular; PSL: Pressure Strain Loop; MW: Myocardial work; GLS: Global longitudinal strain; 2DE: Two-dimensional echocardiography; STE: Speckle tracking echocardiography; HF: Heart failure; BMI: Body mass index; SBP: Systolic blood pressure; DBP: Diastolic blood pressure; HbA1c: Glycosylated hemoglobin; HT: Hypertension; LAD: Left atrial dimension; LVEDD: Left ventricular end-diastolic dimension; LVESD: Left ventricular end-systolic dimension; IVS: Interventricular septum; PWT: Posterior wall thinkness; LVEDV: Left ventricular end-diastolic volume; LVESV: Left ventricular end-systolic volume; LVSV: Left ventricular-stroke volume; LVEF: Left ventricular ejection fraction; A: Late diastolic mitral flow (pulse Doppler); E: Early diastolic mitral flow (pulse Doppler)mitral annulus(tissue Doppler); $\mathrm{e}^{\prime}$ : Average of the peak early diastolic relaxation velocity of the septal and lateral; LVDF: Left ventricular diastolic function; GLS: Left ventricular global
}

longitudinal strain; GWl: Global myocardial work index; GCW: Global constructive work; GWW: Global wasted work; GWE: Global myocardial work efficiency.

\section{Acknowledgements}

Thanks for the support and help of our D. team of ultrasound department Shenzhen People's Hospital. We thank all the investigators and subjects who participated in this project.

\section{Authors' contributions}

LSL, JFX and FJD participated in the study design. LSL, ZMD, JL, YLZ and LXC participated in data collection. LSL, BBS and LXC performed the statistical analysis. LSL drafted the article. All authors read and approved the final manuscript.

\section{Funding}

This project was supported by Natural Science Foundation of Shenzhen, No: JCYJ20190806151807192. The authors declare that the funding body was not involved in study design, data collection, analysis, interpretation and writing of the study.

\section{Availability of data and materials}

The datasets generated and/or analyzed during the current study are not publicly available, but are available from the corresponding author on reasonable request.

\section{Declarations}

\section{Ethics approval and consent to participate}

This study was in accordance with the Declaration of Heksinki and was approved by the medical ethics committee of Shenzhen People's Hospital.

\section{Consent for publication}

Not applicable.

\section{Competing interests}

The authors declare that they have no competing interests.

\section{Author details}

${ }^{1}$ Department of Ultrasound, First Affiliated Hospital of Southern University of Science and Technology, Shenzhen Medical Ultrasound Engineering Center, Shenzhen People's Hospital, 1017 Dongmen North Road, Luohu District, Shenzhen 518020, Guangdong, China. ${ }^{2}$ Department of Ultrasound, Second Clinical College of Jinan University, Shenzhen People's Hospital, Shenzhen, China.

Received: 30 August 2021 Accepted: 29 January 2022

Published online: 17 February 2022

\section{References}

1. Saeedi P, Petersohn I, Salpea P, Malanda B, Karuranga S, Unwin N, Colagiuri S, Guariguata L, Motala AA, Ogurtsova K, et al. Global and regional diabetes prevalence estimates for 2019 and projections for 2030 and 2045 : results from the International Diabetes Federation Diabetes Atlas, 9(th) edition. Diabetes Res Clin Pract. 2019;157:107843.

2. Guariguata L, Whiting DR, Hambleton I, Beagley J, Linnenkamp U, Shaw JE. Global estimates of diabetes prevalence for 2013 and projections for 2035. Diabetes Res Clin Pract. 2014;103(2):137-49.

3. Marwick TH, Ritchie R, Shaw JE, Kaye D. Implications of underlying mechanisms for the recognition and management of diabetic cardiomyopathy. J Am Coll Cardiol. 2018;71(3):339-51.

4. Dei Cas A, Khan SS, Butler J, Mentz RJ, Bonow RO, Avogaro A, Tschoepe D, Doehner W, Greene SJ, Senni M, et al. Impact of diabetes on epidemiology, treatment, and outcomes of patients with heart failure. JACC Heart Fail. 2015;3(2):136-45

5. Torella D, laconetti C, Tarallo R, Marino F, Giurato G, Veneziano C, Aquila I, Scalise M, Mancuso T, Cianflone $E$, et al. miRNA regulation of the hyperproliferative phenotype of vascular smooth muscle cells in diabetes. Diabetes. 2018;67(12):2554-68. 
6. Dunlay SM, Givertz MM, Aguilar D, Allen LA, Chan M, Desai AS, Deswal A, Dickson W, Kosiborod MN, Lekavich CL, et al. Type 2 diabetes mellitus and heart failure: a scientific statement from the American Heart Association and the Heart Failure Society of America: this statement does not represent an update of the 2017 ACC/AHA/HFSA heart failure guideline update. Circulation. 2019;140(7):e294-324.

7. Ledru F, Ducimetière P, Battaglia S, Courbon D, Beverelli F, Guize L, Guermonprez $J$, Diébold B. New diagnostic criteria for diabetes and coronary artery disease: insights from an angiographic study. J Am Coll Cardiol. 2001;37(6):1543-50.

8. Kenny HC, Abel ED. Heart failure in type 2 diabetes mellitus. Circ Res. 2019;124(1):121-41

9. Marfella R, Sasso FC, Cacciapuoti F, Portoghese M, Rizzo MR, Siniscalchi M, Carbonara O, Ferraraccio F, Torella M, Petrella A, et al. Tight glycemic control may increase regenerative potential of myocardium during acute infarction. J Clin Endocrinol Metab. 2012;97(3):933-42.

10. Sasso FC, Rinaldi L, Lascar N, Marrone A, Pafundi PC, Adinolfi LE, Marfella R. Role of tight glycemic control during acute coronary syndrome on CV outcome in type 2 diabetes. J Diabetes Res. 2018;2018:3106056.

11. Fang $Z Y$, Leano $R$, Marwick $T H$. Relationship between longitudinal and radial contractility in subclinical diabetic heart disease. Clin Sci (Lond). 2004;106(1):53-60.

12. Ernande L, Bergerot C, Girerd N, Thibault H, Davidsen ES, Gautier Pignon-Blanc P, Amaz C, Croisille P, De Buyzere ML, Rietzschel ER, et al. Longitudinal myocardial strain alteration is associated with left ventricular remodeling in asymptomatic patients with type 2 diabetes mellitus. J Am Soc Echocardiogr. 2014;27(5):479-88.

13. Hiemstra YL, van der Bijl P, El Mahdiui M, Bax JJ, Delgado V, Marsan NA. Myocardial work in nonobstructive hypertrophic cardiomyopathy: implications for outcome. J Am Soc Echocardiogr. 2020;33(10):1201-8.

14. Russell K, Eriksen M, Aaberge L, Wilhelmsen N, Skulstad H, Remme EW, Haugaa KH, Opdahl A, Fjeld JG, Gjesdal O, et al. A novel clinical method for quantification of regional left ventricular pressure-strain loop area: a non-invasive index of myocardial work. Eur Heart J. 2012;33(6):724-33.

15. Boe E, Skulstad H, Smiseth OA. Myocardial work by echocardiography: a novel method ready for clinical testing. Eur Heart J Cardiovasc Imaging. 2019;20(1):18-20.

16. El Mahdiui M, van der Bijl P, Abou R, Ajmone Marsan N, Delgado V, Bax JJ. Global left ventricular myocardial work efficiency in healthy individuals and patients with cardiovascular disease. J Am Soc Echocardiogr. 2019;32(9):1120-7.

17. Fortuni F, Butcher SC, van der Kley F, Lustosa RP, Karalis I, de Weger A, Priori SG, van der Bijl P, Bax JJ, Delgado V, et al. Left ventricular myocardial work in patients with severe aortic stenosis. J Am Soc Echocardiogr. 2021;34(3):257-66.

18. Recommendations for Cardiac Chamber Quantification by Echocardiography in Adults: An Update from the American Society of Echocardiography and the European Association of, Cardiovascular Imaging. Eur Heart J Cardiovasc Imaging 2016;17(4):412.

19. Manganaro R, Marchetta S, Dulgheru R, llardi F, Sugimoto T, Robinet S, Cimino S, Go YY, Bernard A, Kacharava G, et al. Echocardiographic reference ranges for normal non-invasive myocardial work indices: results from the EACVI NORRE study. Eur Heart J Cardiovasc Imaging. 2019;20(5):582-90.

20. Smiseth OA, Donal E, Penicka M, Sletten OJ. How to measure left ventricular myocardial work by pressure-strain loops. Eur Heart J Cardiovasc Imaging. 2021;22(3):259-61.

21. Strain WD, Paldánius PM. Diabetes, cardiovascular disease and the microcirculation. Cardiovasc Diabetol. 2018;17(1):57.

22. Cole JB, Florez JC. Genetics of diabetes mellitus and diabetes complications. Nat Rev Nephrol. 2020;16(7):377-90.

23. Chan J, Shiino K, Obonyo NG, Hanna J, Chamberlain R, Small A, Scalia IG, Scalia W, Yamada A, Hamilton-Craig CR, et al. Left ventricular global strain analysis by two-dimensional speckle-tracking echocardiography: the learning curve. J Am Soc Echocardiogr. 2017;30(11):1081-90.

24. Pedrizzetti G, Claus P, Kilner PJ, Nagel E. Principles of cardiovascular magnetic resonance feature tracking and echocardiographic speckle tracking for informed clinical use. J Cardiovasc Magn Reson. 2016;18(1):51.

25. Bugger $\mathrm{H}$, Abel ED. Molecular mechanisms of diabetic cardiomyopathy. Diabetologia. 2014;57(4):660-71.
26. Chan J, Edwards NFA, Khandheria BK, Shiino K, Sabapathy S, Anderson B, Chamberlain R, Scalia GM. A new approach to assess myocardial work by non-invasive left ventricular pressure-strain relations in hypertension and dilated cardiomyopathy. Eur Heart J Cardiovasc Imaging. 2019;20(1):31-9.

27. Hubert A, Le Rolle V, Leclercq C, Galli E, Samset E, Casset C, Mabo P, Hernandez A, Donal E. Estimation of myocardial work from pressurestrain loops analysis: an experimental evaluation. Eur Heart J Cardiovasc Imaging. 2018;19(12):1372-9.

28. Russell K, Eriksen M, Aaberge L, Wilhelmsen N, Skulstad H, Gjesdal O, Edvardsen T, Smiseth OA. Assessment of wasted myocardial work: a novel method to quantify energy loss due to uncoordinated left ventricular contractions. Am J Physiol Heart Circ Physiol. 2013;305(7):H996-1003.

29. Manganaro R, Marchetta S, Dulgheru R, Sugimoto T, Tsugu T, llardi F, Cicenia M, Ancion A, Postolache A, Martinez C, et al. Correlation between non-invasive myocardial work indices and main parameters of systolic and diastolic function: results from the EACVI NORRE study. Eur Heart J Cardiovasc Imaging. 2020;21(5):533-41.

30. Boudina S, Abel ED. Diabetic cardiomyopathy revisited. Circulation. 2007;115(25):3213-23.

31. Rijzewijk LJ, van der Meer RW, Smit JW, Diamant M, Bax JJ, Hammer S, Romijn JA, de Roos A, Lamb HJ. Myocardial steatosis is an independent predictor of diastolic dysfunction in type 2 diabetes mellitus. J Am Coll Cardiol. 2008;52(22):1793-9.

32. Regan TJ, Wu CF, Yeh CK, Oldewurtel HA, Haider B. Myocardial composition and function in diabetes. The effects of chronic insulin use. Circ Res. 1981;49(6):1268-77.

33. Dillmann WH. Diabetic cardiomyopathy. Circ Res. 2019;124(8):1160-2.

34. Hansen A, Johansson BL, Wahren J, von Bibra H. C-peptide exerts beneficial effects on myocardial blood flow and function in patients with type 1 diabetes. Diabetes. 2002;51(10):3077-82.

35. Batista ANR, Garcia T, Franco EAT, Azevedo PS, Barbosa MF, Zornoff LAM, Minicucci MF, de Paiva SAR, Zucchi JW, de Godoy l, et al. Comparison of morphometry and ventricular function of healthy and smoking young people. BMC Cardiovasc Disord. 2020;20(1):66.

36. Hendriks T, van Dijk R, Alsabaan NA, van der Harst P. Active tobacco smoking impairs cardiac systolic function. Sci Rep. 2020;10(1):6608.

37. Bluemke DA, Kronmal RA, Lima JA, Liu K, Olson J, Burke GL, Folsom AR. The relationship of left ventricular mass and geometry to incident cardiovascular events: the MESA (Multi-Ethnic Study of Atherosclerosis) study. J Am Coll Cardiol. 2008;52(25):2148-55.

38. Yan WF, Gao Y, Zhang Y, Guo YK, Wang J, Jiang L, Li Y, Yang ZG. Impact of type 2 diabetes mellitus on left ventricular diastolic function in patients with essential hypertension: evaluation by volume-time curve of cardiac magnetic resonance. Cardiovasc Diabetol. 2021;20(1):73.

39. Li XM, Jiang L, Guo YK, Ren Y, Han PL, Peng LQ, Shi R, Yan WF, Yang ZG. The additive effects of type 2 diabetes mellitus on left ventricular deformation and myocardial perfusion in essential hypertension: a 3.0 T cardiac magnetic resonance study. Cardiovasc Diabetol. 2020;19(1):161.

40. Chen X, Guo H, Yang Q, Fang J, Kang X. Quantitative evaluation of subclinical left ventricular dysfunction in patients with type 2 diabetes mellitus by three-dimensional echocardiography. Int J Cardiovasc Imaging. 2020;36(7):1311-9.

41. Gulsin GS, Swarbrick DJ, Athithan L, Brady EM, Henson J, Baldry E, Argyridou S, Jaicim NB, Squire G, Walters Y, et al. Effects of low-energy diet or exercise on cardiovascular function in working-age adults with type 2 diabetes: a prospective, randomized, open-label, blinded end point trial. Diabetes Care. 2020;43(6):1300-10.

42. Ikonomidis I, Pavlidis G, Thymis J, Birba D, Kalogeris A, Kousathana F, Kountouri A, Balampanis K, Parissis J, Andreadou I, et al. Effects of glucagon-like peptide-1 receptor agonists, sodium-glucose cotransporter-2 inhibitors, and their combination on endothelial glycocalyx, arterial function, and myocardial work index in patients with type 2 diabetes mellitus after 12-month treatment. J Am Heart Assoc. 2020;9(9):e015716.

\section{Publisher's Note}

Springer Nature remains neutral with regard to jurisdictional claims in published maps and institutional affiliations. 